\title{
Household electricity load disaggregation based on low- resolution smart meter data
}

\author{
Hiroshi Chin, Kenji Tanaka, Rikiya Abe \\ Department of Technology Management for Innovation, Graduate School of Engineering, The University of Tokyo, 214-3,7-3-1 \\ Hongo, Bunkyo, Tokyo, Japan, 113-8656
}

\begin{abstract}
The Japanese government is proceeding to have the electricity companies to install smart meters in residential sectors. Although power companies are installing smart meters in residential areas, electricity data analysis methods for smart meters are not developed in Japan enough. This research shows an analysis method of electricity disaggregation on low-resolution smart meter data to reveal the point of energy saving for the residents. Specifically, we provided that three kinds of disaggregation methods for the disaggregation of Low-resolution data. 1) Two-states-disaggregation, which can separate the active and the inactive state. 2) Three-states-disaggregation, which can separate the load for active, mid-active and inactive states, which can reveal the life pattern specifically. 3) Temperature-sensitive load disaggregation that can separate the total load and temperature-sensitive load. Finally, we also demonstrated an example of how to take advantage of the disaggregation that helps the users to analyse and enhance energy saving.
\end{abstract}

Keywords: Electricity load disaggregation, smart meter data, hidden markov model, energy saving, temperaturesensitive load disaggregation

\section{Introduction}

In recent years, the introduction of smart meters has been actively conducted in various countries. The Japanese government is proceeding to have the electricity companies install smart meters in residential sectors for the purpose of increasing the efficiency of energy-saving and demand-response programs. After the installation of an electric smart meter, it is expected that a large amount of data can be fully utilized to provide valuable, unique services. To achieve further energy-saving advice, the research on the electric data usage has just started in Japan [1].

In particular, the visualization and energy-saving diagnostic services such as electricity disaggregation based on smart meters have earned greater attention. Electricity disaggregation is a process for deducing the life patterns and usage of appliances by detecting and identifying changes in the voltage, current, and amount of power going through a house. The type of electricity disaggregation could be classified by the power-measuring interval of data [2]. K. Armel et al. indicated a summary of patterns across existing electricity disaggregation work. A data frequency of 1 hour -15 min. uses data features such as duration and time of appliance use and can differentiate three general categories: loads that correlate with outdoor temperature, loads that are continuous, and loads that are time-dependent. A 1 min-1 second data frequency starts to be able to differentiate each appliance and the top 10 appliance types: refrigerator, ACs, heaters, pool pumps, washers, dryers, etc. based on the information of steady state steps/transitions of power [2]. As the data frequency gets finer, the capacity of the appliance recognition rises. Contrary to the various possibilities of the electricity disaggregation-based high-resolution smart meter, in Japan, the main electricity companies have decided to install the smart meter with 30 minutes as the measuring

\footnotetext{
* Manuscript received March 10, 2016; revised July 30, 2016.

Corresponding author. Tel.: +81-8066311907; E-mail address: hiroshi.chin@ gmail.com.

doi: $10.12720 /$ sgce $.5 .3 .188-195$
} 
interval for households. Also, it is general to use smart meters based on a sample value with a $60 \sim 15$ minute measuring interval in many other countries such as UK, Australia, and USA [3]-[5]. These facts show that in the future, the penetration of a low-resolution measuring (low sampling interval) smart meter will be much higher than the high-resolution smart meter. In previous research, electricity disaggregation using high-time resolution data was widely studied. On the contrary, data analysis methods for smart meters with low time resolution are not sufficiently developed [2]. Furthermore, as the analysis method, the electricity load clustering based on k-means or EM algorithm has been used for the purpose of marketing and grouping [6]-[10] to reveal the patterns and trends in the demander group. However, the clustering of the specific time series of the electricity load has not been researched. Assuming the electricity load as a time series, the hidden Markov model can cluster the load into states. The comparison of electricity usage between residents is effective in energy saving, according to previous research [10][12].

In this research, we propose a method that can extract electricity usage patterns based on the electricity disaggregation of low-resolution data. There are two kinds of disaggregation. First is based on the electricity load itself; we disaggregate the load based on the distribution and the amount of value. The second is based on the electricity load and the related parameter. Here, we consider the related parameter is out-side temperature, and disaggregated the electricity load based on it. Specifically, we used a hidden Markov model to determine the state of the electricity usage [8]. Based on the information in the previous steps, we could analyse the behaviour of each state. The total research has much practical applications such as remote life pattern diagnosis, life pattern abnormal detection, in-house or off-house suggestion for the delivery service or targeted advice or demand response programs intended to reduce the demand of customers and make effective comparisons among electricity users.

\section{Methods}

\subsection{Two-states-disaggregation}

A Hidden Markov model is a tool for representing probability distributions over sequences of observations [13]. Here, we have two rough steps; the first step is to calculate the hidden states for the time series data that was generated by the smart meter. The second step is to analyse and compare the disaggregated data so that it is possible to provide targeted energy-saving advice to a particular state. Also, comparing the time series of the residents is valid in energy saving, according to previous research [9]. Furthermore, we introduce the average electricity usage of the analysis. The point for this kind of clustering is that it is different from the typical clustering method because it is possible to separate and cluster the time series, depending on the pattern of the different time series. Furthermore, We can introduce the way to separate the load for the state to more than three states to help the demander to identify the load patterns and life patterns. We performed the disaggregation for more than three hidden statuses. Here, the status can indicate the certain life activity such as sleeping, breakfast, working and so on.

\subsection{Hidden markov model}

When the hidden states $(A, B, C .$.$) are defined as Q:=\left\{q_{1}, q_{2}, q_{3} \ldots q_{n}\right\},(1)$ is the posterior probability to have the observation: $=\left\{\mathrm{y}_{1}, \mathrm{y}_{2}, \mathrm{y}_{3}, \ldots \mathrm{y}_{\mathrm{n}}\right\}$, and it can be defined as below.

$$
P(Y \mid Q)=\pi_{q_{0}} \prod_{t=1}^{n} a_{q_{t} q_{t+1}} b_{q_{t}}\left(y_{t}\right)
$$

where,

$$
\begin{aligned}
& \pi_{q_{0}}=\text { The initial emission parameter in the state } \mathrm{q}_{0} . \\
& b_{q_{t}}\left(y_{t}\right)=\text { Emission parameter for an observation associated with state } \mathrm{q}_{\mathrm{t}} .
\end{aligned}
$$


$a_{q_{t} q_{t+1}}=$ Probability of transition from state $\mathrm{q}_{\mathrm{t}}$.

Hidden Markov model can estimate the hidden status by Baum-Welch algorithm and Viterbi algorithm. Baum-Welch algorithm is used to find the each parameter including the initial parameter distribution, the transition distribution and described in the above Viterbi algorithm is a dynamic programming algorithm for finding the most likely hidden states for the coming the test sequence. The difference between the Hidden Markov model and another clustering model is that the Hidden Markov model is targeting the time-series to find the hidden states for each input data. Specially, the pervious time series route will be considered in the decision process of the hidden status so that the distribution of the time series will be considered. Here, we consider the electricity load (30 minutes) as input data, try to find the suitable hidden state based on the emission parameter and probability of transition. The disaggregated hidden states cannot represent some specific appliance, but it is possible to show the certain state that have particular distribution and value. We call this hidden state life pattern. Life pattern can stand for active or in-active state or more specific pattern.

\subsection{Temperature sensitive disaggregation}

In Japan, the temperature sensitive load is consisting of around 30\% of the total load of household [14]. Also, once the control device of the temperature load has been reported it can reduce the house energy consumption around $20 \%$ in the USA. It means that to indicate the approximate temperature sensitive load has the potential to help the demanded to perform the energy saving. We performed the disaggregation for the temperature sensitive load; the status can indicate the life activity that is sensitive to the temperature. The temperature sensitive load can be calculated by the procedure below. The basic idea of temperature sensitive disaggregation is to take advantage of the load gap between the time (season) that is not temperature sensitive and the time (season) is temperature sensitive. We consider the gap is the temperature sensitive.

1) Build a model between average temperature per day and average electricity load per day based on more than one-year data. It can be represented in the Quadratic equation form (The case of electricity load in Japan).

$$
Y_{\text {load }}=T^{2}+T+c, \text { where } T \text { is the average temperature per day }
$$

2) Calculate the lowest average load in a year, set it as $Y_{\text {low }}$, and calculate the ideal load for each step $\left(Y_{\text {ideal }, t}\right)$ for the temperature in that step $(t)$ based on the equation (2).

3) Calculate the $Y_{\text {gap }}$ between ideal load $\left(Y_{\text {ideal, },}\right)$ and lowest load $\left(Y_{\text {low }}\right.$.

4) Calculate the temperature sensitive load $Y_{\text {temp,t }}$ for each step(t) based on the equation(3)

$$
Y_{\text {temp }, t}=Y_{a c t u a l, t} / Y_{\text {ideal }, t} * Y_{\text {gap }, t}\left(Y_{\text {gap }, t}>=0\right)
$$

\section{Case Study}

\subsection{Two-state-disaggregation}

We used the data of a condominium in Tokyo area in Japan. The term of data is one year (2013/1/1 2013/12/31), and the time step of data is 48 steps per day (1 step is 30 minutes). The number of electricity demandedd in the condominium is 75 . We performed the calculation of the hidden state of the time series. Fig. 1 shows the result of disaggregation based on HMM. We consider that there are two states in the time series. One state is the resident has active behaviour (active state); the other state is that the resident has inactive behaviour (inactive state). It shows that the two states could be clearly separated (The darker part indicates the inactive pattern, and the light part indicates the active). In the contrast, Fig. 2 indicates the result of disaggregation based on the K-mans method that is a general method for the 
grouping and clustering for the electricity load. Here, we can find that the k-means method could not classify the status of the active and inactive state. Also, the one advantage of using the Hidden Markov model is that the Hidden Markov model can take the duration of loads into consideration and do not have to make criteria for each time series. For instance, in Fig. 1, the cycle part was not selected for an inactive state because of the fluctuation of the load.

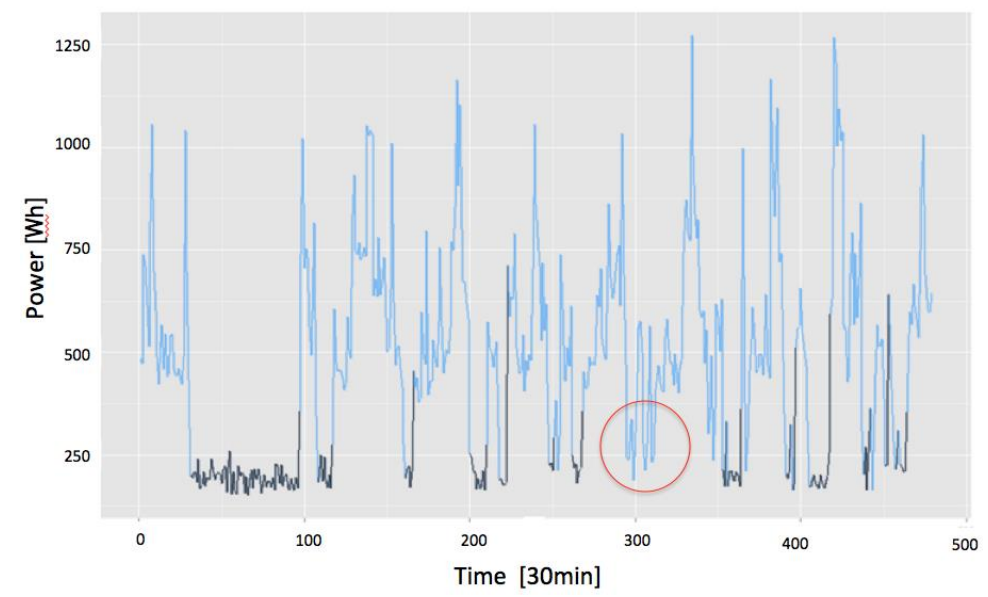

Fig. 1. The result of disaggregation (HMM).

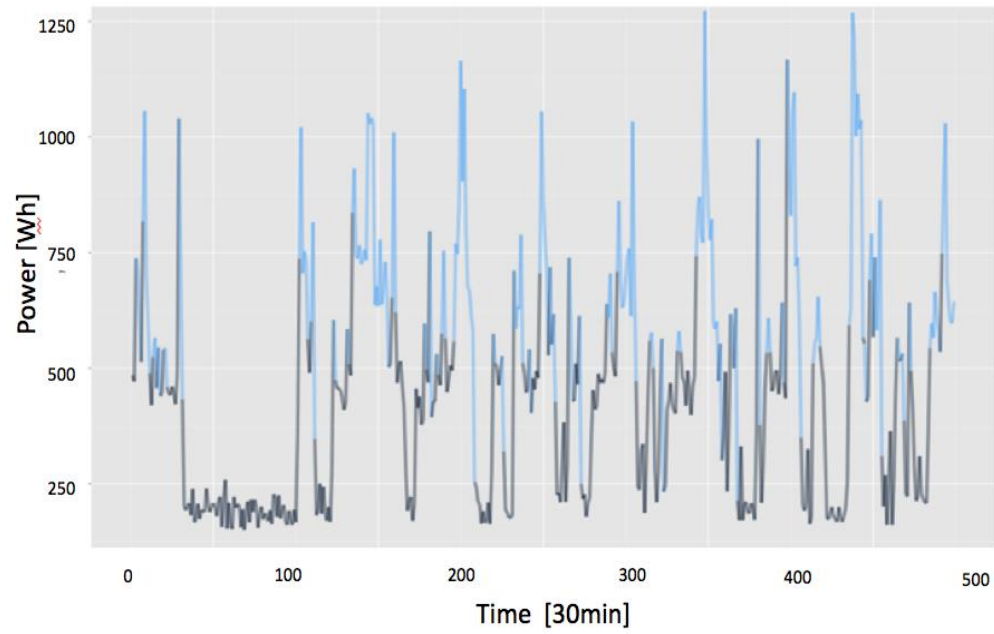

Fig. 2. The result of disaggregation (k-means).

\subsection{Three-states-disaggregation}

Fig. 3 could separate the life pattern to three states (active, mid-active, in-active). Fig. 4 and 5 show the percentage of each status for each period of a demander. The horizontal axis is the time, and the vertical axis illustrates the number of the count for the status (in 10 days). From Fig. 4 and 5, we can find, intuitively, that the inactive status is concentrated in the nighttime and the daytime for this demander, and the most active status is located in the morning and evening. Furthermore, we can understand that, even though the inactive status has a great percentage of electricity usage, the amounts of active and mid-active statuses have much larger electricity usage. It is possible to suggest electricity reduction from the midactive and active statuses. As practical usage, we can take a questionnaire from the demander to know the actual activity and the appliance that is working in that state, or define more states that represent the life patterns 


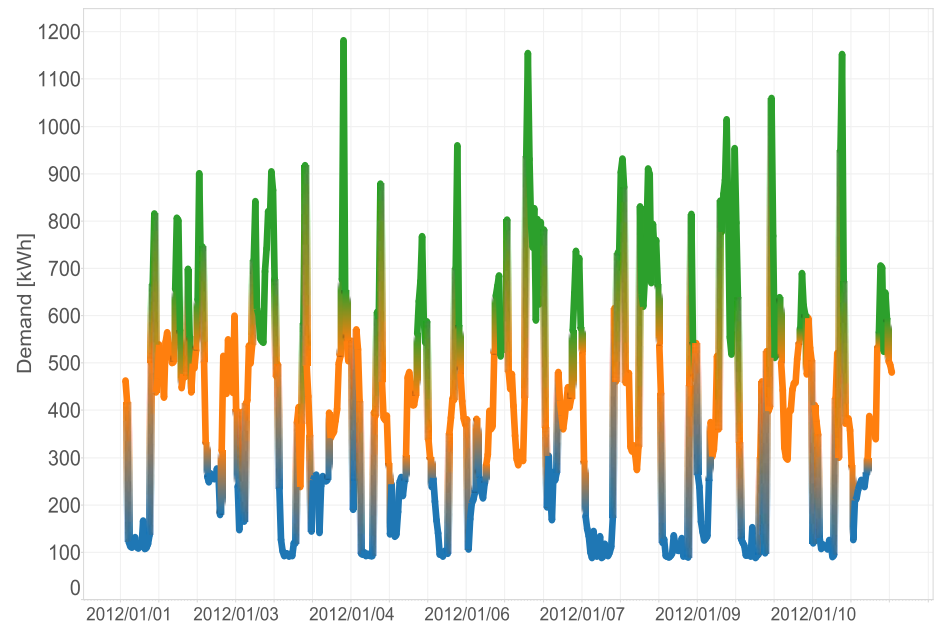

Fig. 3. The result of disaggregation (3 hidden status).

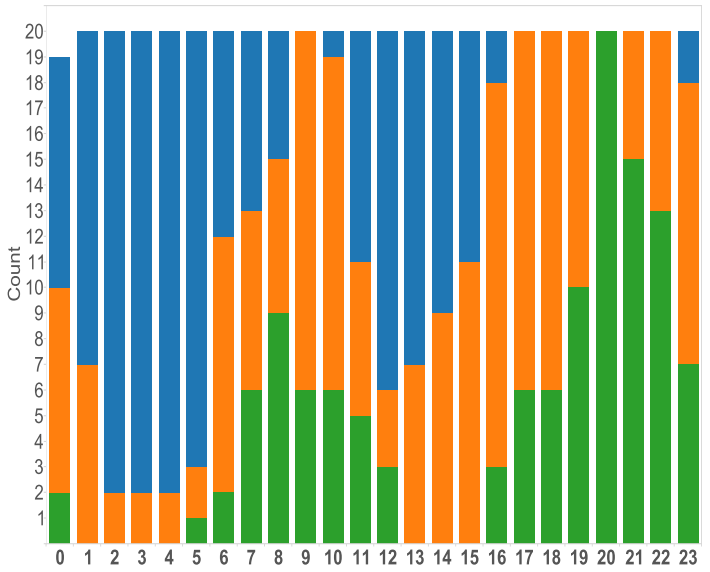

Fig. 4. The percentage of each status for each time of a demander.

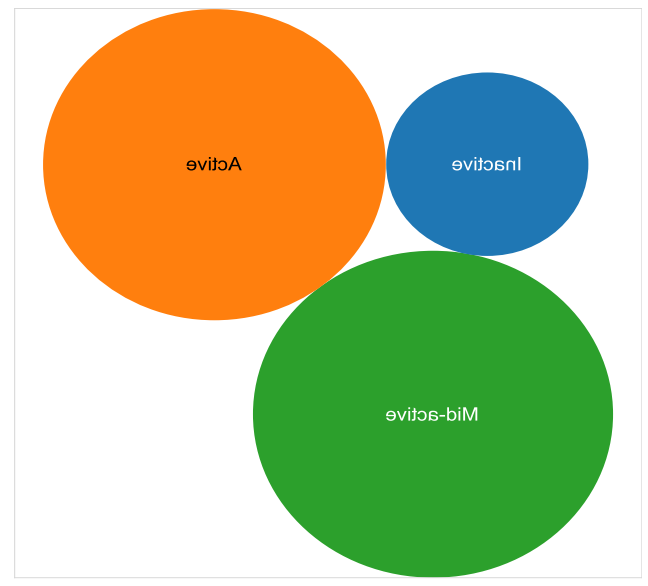

Fig. 5. The total amount of each status.

\subsection{Temperature sensitive load disaggregation}

Following what has been indicated in Section 2.3, the data is from January 2012 to November 2012 for 
a demander in Tokyo. Fig. 7 shows the disaggregation of the temperature sensitive load from the total load. The green graph is the full load of the demander from September to November. The disaggregation is performed based on the model made from Fig. 6. We can find a quadratic relationship between the average temperature and average demand. The trend line was represented by the equation $1.5 *$ temperature ${ }^{2}-50.678 *$ temperature +673 . The $\mathrm{P}$ value was $<0.0001$. In Fig. 6 , we can find that the usage of load is high when the average temperature is over $25^{\circ} \mathrm{C}$ and below $10^{\circ} \mathrm{C}$ (Tokyo). Also, we can tell it is hard to distinguish the load from the total load without the disaggregation. Taking advantage of this method, we can further precisely advise people to reduce the power usage for demand response and power saving.

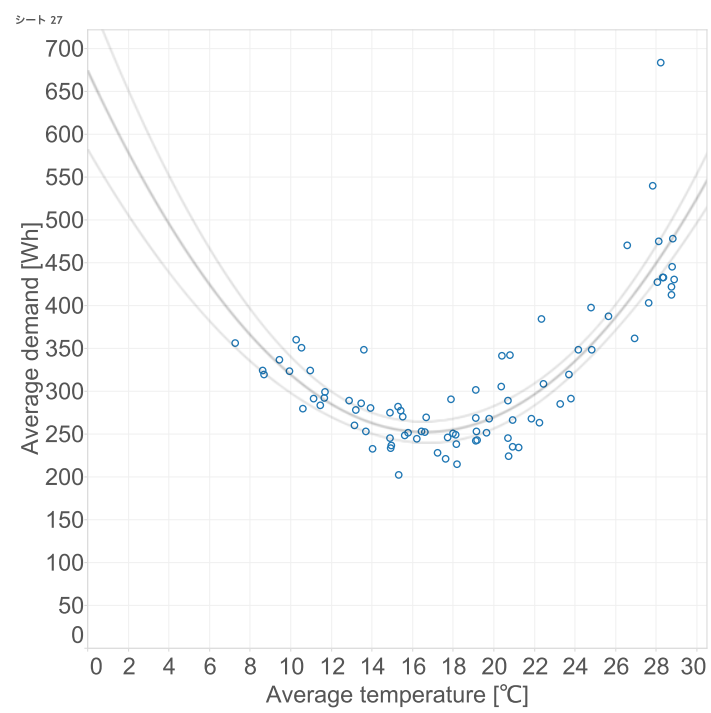

Fig. 6 The plot between average temperature and average demand.

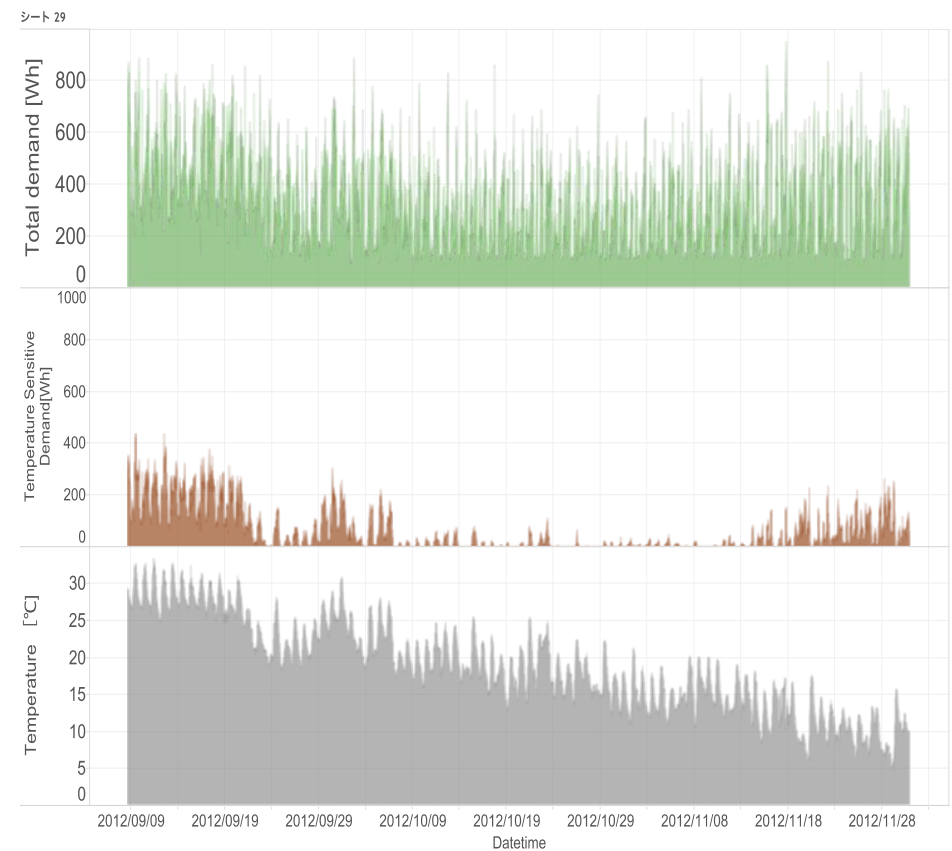

Fig. 7 The disaggregation of the temperature sensitive load (Green (Upper): Total load, Red (Mid): Disaggregated load, Gray (Down): Temperature). 


\subsection{Example for the disaggregated load data analysis}

We performed the analysis of the disaggregated data and had a comparison of the result. Fig. 8 and 9 show the comparison of the electricity usage of rooms in the condominium. The horizontal axis is for the average of the state condition ( 1 stands for active status, 2 stands for inactive status). The vertical axis is for the average electricity usage per day. We can classify the two-dimensional graph to four domains to characterize the demanders as indicated in Fig. 8. Primarily, we can estimate that there is a high potential to perform the energy saving for the room to improve the domain (right upper side) because we assume that these demanders use much electricity even though they are in an inactive state. Fig. 9 indicates the comparison of the electricity usage of two rooms every day for a year, and it suggests that there is more potential to reduce the electricity for the demander (with large dot). The reason is that the average electricity usage of the user demander (with large dot) has a radical change in the mean of the state condition. Also, there is a high variance in electricity usage for the active state of the user demander (with large dot) so that we can assume there is room to improve the electricity usage. All of this analysis can make a system that to provide the information.

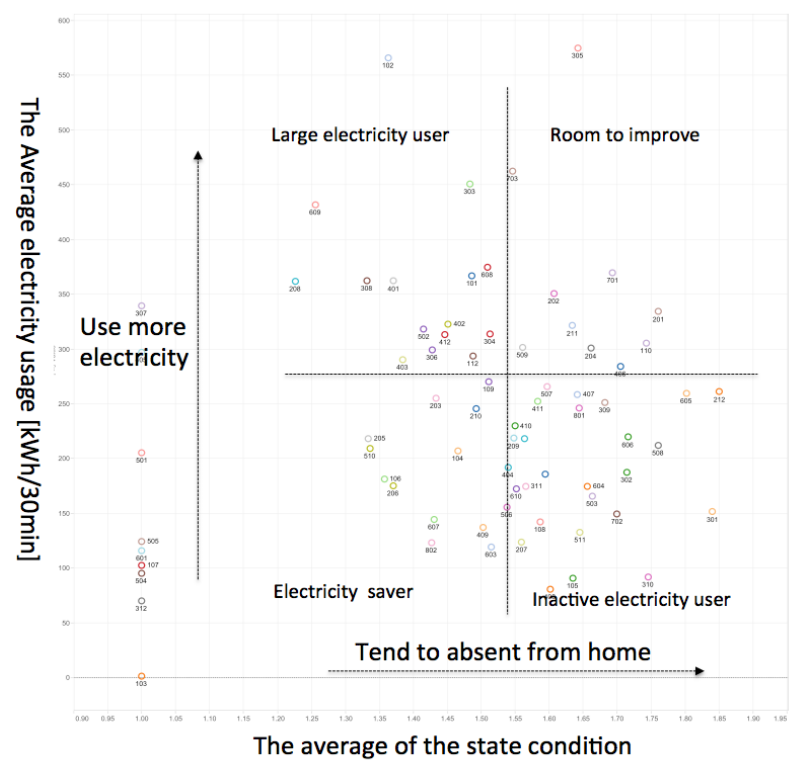

Fig. 8. The comparison of the electricity usage of rooms.

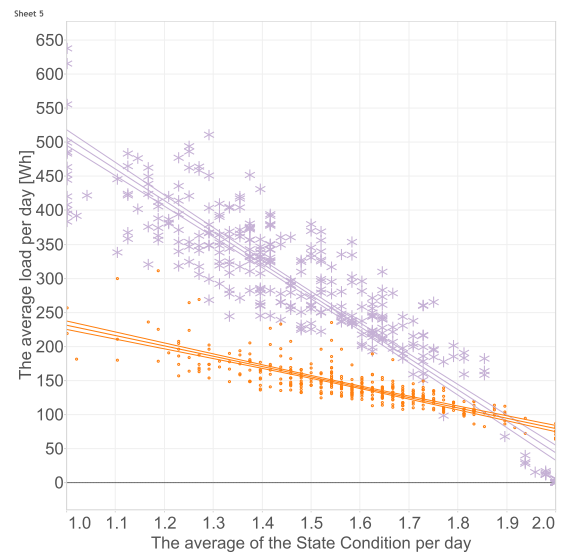

Fig. 9. The comparison of electricity usage of 2 demanders (The average of the state condition versus the average electricity usage). 


\section{Conclusion}

The paper shows an analysis method of electricity disaggregation on low-resolution smart meter data to reveal more precise personalized insights and analysis for energy saving. The result of the disaggregation can be applied to a number of service such as remote life pattern diagnosis, life pattern abnormal detection, in-house or off-house suggestion for the delivery service or targeted advice or demand response programs intended to reduce the demand of customers and make effective comparisons among electricity users. This result is much more feasible than using the finer sampling interval smart meter data because of the popularity of the low-resolution smart meter. As the future perspective, we would like to use the result of disaggregation for the recommendation system for the energy-saving appliance recommendation based on the low-resolution smart meter data.

\section{References}

[1] Komatsu H. Utilization of smart meter data's analytical information - Trend of technology and an exploratory demand analysis. Central Research Institute of Electric Power Industry, Socio-economic Research Center Rep, 2014; No.Y14003.

[2] Armel K, Gupta A, Shrimali G, and Albert A. Is disaggregation the holy grail of energy efficiency? The case of electricity. Energy Policy, 2012; 52:213-234.

[3] Australia Energy Council. (March 2016). [Online]. http://www.energycouncil.com.au/energy_you/what_is_a_smart_meter

[4] Energy UK. (March 2016). [Online]. Available: http://www.energy-uk.org.uk/customers/about-smart-meters/how-much-datais-collected-with-smart-metering-and-is-it-secure.html

[5] Power Energy USA. (March 2016). [Online]. Available: http://www.powerenergyusa.com/demand-response/

[6] Chicco G, Napoli R, Postolache P, Scutariu M, Toader C. Customer characterization options for improving the tariff offer. IEEE Trans. Power Syst., 2003; 18(1):81-387.

[7] Christoph F, David N,Tobias C, et al. Cluster analysis of smart metering data - an implementation in Practice. Business \& Information Systems Engineering, 2012; 4(1):31-39.

[8] Moore. K-means and hierarchical clustering. Stat. Data Min. Tutorials, 2001; 1-24.

[9] McLachlan GJ, Krishnan T. The EM algorithm and extensions. New York, 1997; 359.

[10] Kaile Z, Shanlin Y, Chao S. A review of electric load classification in smart grid environment. Renewable and Sustainable Energy Reviews, 2013; 24:103-110.

[11] Hartman B, Leblanc W. Smart meters, big data, and customer engagement: in pursuit of the perfect portal. In: Proc. of ACEEE Summer Study on Energy Efficiency in Buildings, 2014; 172-182.

[12] Laskey. (March 2016). How behavioral science can lower your energy bill, TED. [Online]. Available: https://www.ted.com/talks/alex_laskey_how_behavioral_science_can_lower_your_energy_bill/transcript

[13] Eddy SR. What is a hidden Markov model? Nat. Biotechnol., 2004; 22(10):1315-1316.

[14] The Federation of Electric power companies of Japan. (March 2016). [Online]. Available: http://www.fepc.or.jp/index.html 